\title{
Quantum gas microscopy: in-situ imaging of ultracold atomic gases
}

In situ Observation of incompressible Mott-insulating domains in ultracold atomic gases

N. Gemelke, X. Zhang, C.-L. Hung, C. Chin

arXiv:0904.1532; Nature 460, 995 (2009)

Probing the Superfluid-to-Mott-Insulator Transition at the Single-Atom Level W. S. Bakr, A. Peng, M. E. Tai, R. Ma, J. Simon, J. I. Gillen, S. Fölling, L. Pollet, and M. Greiner.

arXiv:1006.0754; Science 329, 547 (2010)

Single-Atom Resolved Fluorescence Imaging of an Atomic Mott Insulator J.F. Sherson, C. Weitenberg, M. Endres, M. Cheneau, I. Bloch, S. Kuhr arXiv:1006.3799; Nature advance online publication 18 August 2010 — doi:10.1038/nature09378

\section{Recommended with a Commentary by Matthias Troyer, ETH Zürich}

Ultracold atomic quantum gases offer a flexible toolbox for the clean and controlled realization of strongly correlated quantum systems. Superimposing an optical lattice made from standing waves of laser light onto a quantum degenerate Bose gas, as originally proposed by Jaksch et al. [Phys. Rev. Lett. 81, 3108 (1998], results in a clean experimental realization of bosonic Hubbard models in which the coupling strength $U$, hopping amplitudes $t_{i j}$ and even the dimensionality can be tuned and even dynamically changed during the experiment. Replacing the Bose gas by a cold Fermi gas similarly realizes a fermionic Hubbard model and even multi-component gases and mixtures can be realized. The interest in these optical lattice emulators arises from the potential to study exotic phases of the Hubbard model and related models - whose physics still escapes our theoretical understanding in the most interesting regions of their phase diagrams - through controlled experiments on a clean realization of the model in an experiment.

Following the seminal first experimental realization of such an optical lattice system and the observation of a superfluid to Mott insulator by Greiner et al. [Nature 415, 39 (2002)] there has been an explosive growth in this field and the subsequent realization of bosonic, fermionic, and mixtures, in one, two and three dimensions with different lattice structures. An important milestone towards the use of these optical lattice emulators as tools to investigate the unknown physics of Hubbard models has been the detailed comparison of experiments on a Bose-Hubbard model with large-scale ab-initio numerical simulations of the same model by S. Trotzky, L. Pollet et al. [arXiv:0905.4882, to appear in Nature Physics].

A limitation of most current experiments is that the ultracold quantum gas has to be trapped in a magnetic or optical trap, leading to a (usually) 
harmonic confinement potential and thus an inhomogeneous chemical potential $\mu_{i}$. Most probes of the system, such as the time-of-flight images that essentially record the momentum distribution of the gas after its release from the trap, probe the whole system and thus average over regions with different density, and potentially different phases.

The three papers recommended here describe an important step away from this limitation, by presenting experimental setups in which in-situ imaging of bosonic atoms in a two-dimensional optical lattice with single atom resolution is shown to be possible. By directly recording real-space images of the locations of the atoms it is now possible to locally probe the system in a specific phase, and to directly observe, e.g. a Mott insulator with a density of one atom per site, surrounded by a superfluid or normal fluid with lower density, as shown in the figure:

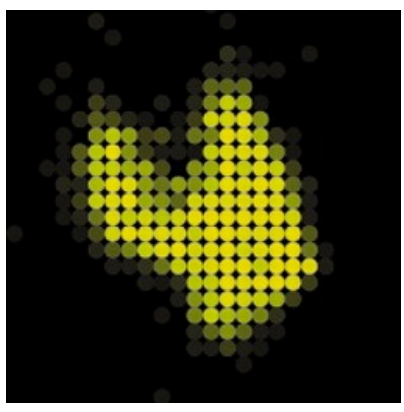

and one can even see the atoms move over time as demonstrated in movies on Markus Greiner's web page http://greiner. physics .harvard.edu/QGM.html

While experimental details of the optical lattice setup and the imaging system differs among the three papers, the basic idea is that of an optical microscope directly observing a two-dimensional quantum gas loaded into an optical lattice. To give a few more details I will use the Harvard experiment of Markus Greiner's group as an example. They trap a few thousand Rubidium atoms in a single narrow well of a standing wave just $9 \mu \mathrm{m}$ from an in-vacuum lens. This lens is part of an optical imaging system with a resolution of about $600 \mathrm{~nm}$, fine enough to resolve single atoms in a lattice with $680 \mathrm{~nm}$ lattice spacing with a high fidelity.

The lattice is ingeniously created by using the microscope not only to observe the atoms, but also to project an optical square lattice potential onto the quantum gas! Optical lattice experiments on quantum gases are already on the forefront of quantum optics research and require a lot of experimental expertise and skill. Adding the imaging system of an optical microscope capable of seeing individual atoms is a feat of optical engineering.

These three papers have opened the door to a new generation of experiments on ultracold quantum gases and we can look forward to exciting new results. What can one do with these microscopes? The possibilities are manyfold. Besides directly seeing a Mott insulator, the images allow in-situ measurements of 
the occupation number distribution in the Mott insulating, superfluid and normal fluid phases. Measuring the fluctuations enables a direct determination of the temperature [Q. Zhou and T.-L. Ho, arXiv:0908.3015v2 and P.N. Ma et al., arXiv:1007.3529]. Observing the time dependence of the density distribution allows the "microscopic" study of non-equilibrium phenomena and thermalization in strongly interacting quantum many body systems. In addition such microscopes can not only be used to observe the atoms, but can also be used to focus a time dependent optical lattice pattern onto the quantum gas, allowing for almost arbitrarily shaped time-dependent lattices! One has barely scratched the surface of what is possible with these quantum gas microscopes. They will certainly be instrumental in furthering our understanding of equilibrium and non-equilibrium properties of strongly correlated quantum systems. 